\title{
Comparison of diurnal blood pressure and urine production between people with and without chronic spinal cord injury
}

\author{
Min Yin Goh ${ }^{1,2,3} \cdot$ Melinda S. Millard ${ }^{1,4} \cdot$ Edmund C. K. Wong ${ }^{1}$ - David J. Berlowitz $\mathbb{D}^{2,5,6} \cdot$ Marnie Graco ${ }^{2,5}$. \\ Rachel M. Schembri ${ }^{5}$. Douglas J. Brown ${ }^{3}$. Albert G. Frauman ${ }^{1,2}$. Christopher J. O'Callaghan ${ }^{1,2}$
}

Received: 22 September 2017 / Revised: 8 February 2018 / Accepted: 12 February 2018 / Published online: 2 March 2018

(C) International Spinal Cord Society 2018

\begin{abstract}
Study design Observational study.

Objectives To quantify diurnal blood pressure (BP) patterns and nocturnal hypertension and to measure diurnal urine production in people with chronic spinal cord injury (SCI), compared with controls without SCI.

Setting Chronic SCI population in the community in Victoria, Australia.

Methods Participants were recruited by advertisement, and sustained SCI at least a year prior or were healthy able-bodied volunteers. Participants underwent ambulatory BP monitoring (ABPM), measurement of urine production, and completed questionnaires regarding orthostatic symptoms. Comparisons were made between participants with tetraplegia or paraplegia and able-bodied controls. Participants with night:day systolic BP $<90 \%$ were classified as dippers, $90-100 \%$ as nondippers, and $>100 \%$ as reverse dippers.

Results Groups with tetraplegia $(n=51)$ and paraplegia $(n=33)$ were older $(42.1 \pm 15$ and $41.1 \pm 15$ vs. $32.4 \pm 13$ years, mean \pm s.d.) and had a higher prevalence of males ( 88 and $85 \%$ vs. $60 \%)$ than controls $(n=52)$. The average BP was 110.8 $\pm 1.5 / 64.4 \pm 1.2 \mathrm{mmHg}, 119.4 \pm 2.1 / 69.8 \pm 1.5 \mathrm{mmHg}$, and $118.1 \pm 1.4 / 69.8 \pm 1.0 \mathrm{mmHg}$ in tetraplegia, paraplegia, and controls, respectively. Of participants with tetraplegia, paraplegia and controls, reverse dipping was observed in 45,13 , and $2 \%(p<0.001)$, while nocturnal hypertension was observed in 13,23 , and $18 \%$, respectively $(p=0.48)$. A reduction in nocturnal urine flow rate compared with the day was observed in paraplegia and controls, but not tetraplegia.

Conclusions Similar to the effects of acute SCI, chronic SCI, specifically tetraplegia, also causes isolated nocturnal hypertension, reverse dipping, orthostatic intolerance, and nocturnal polyuria. Cardiovascular risk management and assessment of orthostatic symptoms should include ABPM.
\end{abstract}

Electronic supplementary material The online version of this article (https://doi.org/10.1038/s41393-018-0081-3) contains supplementary material, which is available to authorised users.

Min Yin Goh

Min.Goh@austin.org.au

1 Department of Clinical Pharmacology, Austin Hospital, Heidelberg, VIC, Australia

2 Department of Medicine, University of Melbourne, Parkville, VIC, Australia

3 The Spinal Research Institute, Kew, VIC, Australia

4 Victorian Spinal Cord Service, Heidelberg, VIC, Australia

5 Institute for Breathing and Sleep, Austin Hospital, Heidelberg, VIC, Australia

6 Department of Respiratory and Sleep Medicine, Austin Hospital, Heidelberg, VIC, Australia

\section{Introduction}

Blood pressure (BP) control is dramatically impaired by disruption of the neural pathways that regulate cardiovascular function. The clinical consequences of autonomic damage in spinal cord injury (SCI) are well recognised and include severe hypotension and persistent bradycardia, large daily fluctuations in BP, autonomic dysreflexia and potentially premature arterial disease, and increased cardiovascular risk [1-3].

Autonomic disease in the able-bodied population produces similarly severe clinical impairment. In this cohort, measurement of BP during ambulation has proven invaluable in the management of symptoms, control of cardiovascular risk, and identification of potential mechanisms which may contribute to orthostatic intolerance. In particular, ambulatory $\mathrm{BP}$ monitoring $(\mathrm{ABPM})$ has been instrumental in identifying 
the disruptions of diurnal variation of BP and the role that elevated nocturnal pressures may play in nocturnal diuresis and natriuresis-which have been proposed as contributors to postural hypotension $[4,5]$.

Using ambulatory monitoring, we recently documented the temporal changes in BP and urine production over the year following SCI [3]. Compared with control participants, SCI was characterised by high rates of nocturnal hypertension, reversal of diurnal variation of BP, and absent diurnal variation in urine production, especially in people with tetraplegia. Whether these effects persist beyond the first 12 months following SCI, and the extent to which people with chronic SCI are affected by them, remains unknown. In this study, we examined the diurnal BP, diurnal urine production, and orthostatic symptoms in people with chronic SCI, and compared them with that of controls.

\section{Methods}

Participants were recruited prospectively by advertisement within the hospital and in the community through general and SCI-specific services. Previous patients of the hospital were also invited by letters and telephone calls. Participants with SCI had traumatic tetraplegia or paraplegia with a duration of injury of at least 1 year, regardless of concurrent health conditions. Able-bodied volunteers without known cardiovascular conditions were recruited through advertisements in the hospital and surrounding universities.

Participants underwent an interview, and medical records were reviewed, to characterise demographic details (age, gender) and details of SCI (date, mechanism of injury, level, and completeness of injury [6]), medical conditions, and concurrent therapy. Participants were assigned the tetraplegia group if their lesion was in the cervical region, and the paraplegia group if their lesion was in the thoracic, lumbar, or sacral region. Those with paraplegia were further classified as high paraplegia if the lesion was between $\mathrm{T} 1$ and T6 or as low paraplegia if the lesion was below T6 [6].

\section{Ambulatory BP monitoring}

Ambulatory BP measurements were obtained using the Card (X)plore monitor (Meditech, Budapest, Hungary), with a cuff of appropriate size, worn by each participant over about $24 \mathrm{~h}$ in an outpatient setting. Measurements were taken halfhourly during the day (0600-2200 h) and hourly at night (2200-0600 h). Results were analysed according to mean 24$\mathrm{h}$, day $(1000-2000 \mathrm{~h})$, and night $(0000-0600 \mathrm{~h})$ values for systolic BP (SBP), diastolic BP (DBP), and pulse rate. Day and night were defined by narrow fixed clock-time intervals to eliminate morning and evening transition periods which are associated with considerable variation in $\mathrm{BP}$ [7] and in postural change among groups. These correspond well with actual awake and asleep periods [8]. This is especially applicable to the SCI population who may dress in bed after getting upright for the morning routine and may return to bed for a duration before going to sleep for the night, hence there is a period over which postural change occurs.

As previously described, the extent of nocturnal dip in BP was calculated as the ratio of night SBP to day SBP, expressed as a percentage (night:day SBP). Participants with a night:day SBP of $<90 \%$ (the normal pattern) were classified as "dippers", 90-100\% as "nondippers", and >100\% as "reverse dippers" [9]. Hypertension was defined as average 24-h BP greater than $130 / 80 \mathrm{mmHg}$, day hypertension as average day BP above $135 / 85 \mathrm{mmHg}$, and nocturnal hypertension as average night BP above $120 / 70 \mathrm{mmHg}$ [10].

\section{Postural BP measurement}

Postural BP was measured upon arising from bed on the morning after the monitor was applied. Participants or their carers took manual measurements from the ambulatory device at 1-min intervals. The participants were required to be recumbent for the first two measurements and then standing (seated if participants were unable to stand) for the next three measurements. Orthostatic hypotension was defined as a fall in SBP of $20 \mathrm{mmHg}$ or DBP of $10 \mathrm{mmHg}$ within $3 \mathrm{~min}$ of assuming the upright position [11].

\section{Urine measurements}

Urine output was recorded over 3 consecutive days, generally within a week of BP monitoring. Participants were instructed to record the time that they voided or emptied their catheter bag on waking on the first day (Day 1), and then to record urine volume and time at each void or when the catheter bag was emptied, until and including the measurement on first waking on Day 4. A measuring jug was provided for measurement of voided urine or urine emptied from the catheter bag. For the purposes of this study, the terms urine output and urine production are used interchangeably and refer to the volume of urine measured from voiding or emptying of the catheter bag. The day and night rates of urine output were calculated by dividing the volume of urine by the time between measurements and expressed as $\mathrm{ml} / \mathrm{min}$. Measurements reported were average values for the 3 days and nights. For each group, comparisons were made between the rate of urine output during the day and the night.

\section{Symptom assessment}

Participants were asked to rate their symptoms of orthostatic intolerance over the preceding week on a scale of 0 for none 
to 10 for the worst possible. Symptoms of orthostatic intolerance were explained as symptoms (lightheadedness, dizziness) pertaining to low BP often arising from postural change or remaining upright for some time, and relieved with tilting backwards, lifting legs, or lying down.

\section{Study groups}

The main aim was to compare tetraplegia with paraplegia and with healthy able-bodied volunteers. Additionally, high paraplegia was compared with low paraplegia, and complete with incomplete tetraplegia and paraplegia. For participants with SCI, urine production and orthostatic symptoms were compared across the three categories of diurnal BP variation-dipping, nondipping, and reverse dipping. This comparison was made for participants with: (a) SCI, (b) complete SCI, and (c) tetraplegia.

\section{Statistical analysis}

Analysis was performed using Microsoft Excel (Microsoft, Redmond, WA, USA) and R software (http://www.rproject.org). Participants' data was included for analysis if they had complete measurements for at least one of ABPM or urine diary. Factorial ANCOVA was used to compare continuous variables between study groups (such as BP), adjusting for gender and age; Tukey's Honestly Significant Difference tests were used for pairwise comparisons where there was a significant difference between groups. Paired $t$ tests were used to compare the day and night continuous variables (pulse rate and urine output rates) within each group. The chi-square test was used for categorical variables (such as gender and dipping status). The Kruskal-Wallis test was used for ordinal, non-parametric data (symptom rating scale). Significance was set at $p<0.05$. Range intervals represent standard error of the mean (s.e.m.).

\section{Statement of ethics}

We certify that all applicable institutional and governmental regulations concerning the ethical use of human volunteers were followed during the course of this research. The protocol for this study was approved by the Human Research Ethics Committee of Austin Health.

\section{Results}

\section{Study group characteristics}

Participants were predominantly male and middle aged (Table 1). There were significantly more males in the SCI groups than in the able-bodied group, and participants in the SCI groups were older (Table 1).

Eight participants (three with tetraplegia, five with paraplegia) were taking anti-hypertensive medications and two (both with tetraplegia) were on pressor agents (fludrocortisone, pseudoephedrine, and liquorice root). None of the control participants were taking medications that affect BP.

ABPM data was incomplete for 4, 3, and 1 participant(s) in the tetraplegia, paraplegia, and control groups, respectively, while urine measurements were incomplete in 20, 9, and 10, respectively. This was due to insufficient readings (ABPM), inability to tolerate the monitor, or not completing urine measurements.

\section{BP and pulse rates}

The tetraplegia group had lower 24-h and day BP than the paraplegia and control groups, but night BP did not differ between groups (Table 2). Night BP exceeded day BP in $45 \%$ of participants with tetraplegia, but this was the case for only $13 \%$ of those with paraplegia and $2 \%$ of controls. Dippers comprised $26 \%$ of those with tetraplegia, $70 \%$ of those with paraplegia, and $82 \%$ of the controls. In those with elevated night $\mathrm{BP}$, nocturnal hypertension was seen in the absence of day hypertension in 4 of 6 in the tetraplegia group, 5 of 7 in the paraplegia group, and 1 of 9 in the control group.

Participants with tetraplegia had lower $24 \mathrm{~h}$ and daytime pulse rates compared with other groups, while those with paraplegia had higher pulse rates in the day compared with other groups (Table 2). The nocturnal fall in pulse rate was observed in each group.

The differences across groups for SBP and pulse rates were present after adjusting for gender and age.

\section{Subgroups}

\section{High and low paraplegia}

Values for BP and pulse rate in participants with high paraplegia were generally located between those with tetraplegia and low paraplegia (Supplementary Table S1). There were significant differences between high paraplegia and tetraplegia for day pressures, nocturnal dipping, 24-h, and day pulse rates, and no significant differences between high and low paraplegia for BP or pulse rate parameters. Dippers comprised 74 and $65 \%$ of participants with high and low paraplegia, respectively.

\section{Complete and incomplete $\mathrm{SCl}$}

Diurnal BP variation was lost in both complete and incomplete tetraplegia, with night:day SBP being in the 
Table 1 Characteristics of study group

\begin{tabular}{|c|c|c|c|}
\hline & Tetraplegia $(n=51)$ & Paraplegia $(n=33)$ & Controls $(n=52)$ \\
\hline Age (years) & $42.1 \pm 15^{*}$ & $41.1 \pm 15^{*}$ & $32.4 \pm 13$ \\
\hline Median & 41 & 41 & 28 \\
\hline Range & $16-70$ & $22-75$ & $16-65$ \\
\hline Males & $45(88 \%)^{*}$ & $28(85 \%)^{*}$ & $31(60 \%)$ \\
\hline Level of SCI & C2-C8: 51 & $\begin{array}{l}\text { T1-T6: } 17 \\
\text { T7-L1: } 16\end{array}$ & - \\
\hline Duration of SCI (years) & $7.5 \pm 9.3$ & $5.9 \pm 6.4$ & \\
\hline Median & 4 & 3 & \\
\hline Range & $1-51$ & $1-28$ & \\
\hline Complete/incomplete & $21 / 30$ & $24 / 9$ & - \\
\hline Neurological & $\mathrm{C} 1-4, \mathrm{ABC}: 10$ & T1-6, ABC: 17 & \\
\hline Classification & $\begin{array}{l}\text { C5-8, ABC: } 32 \\
\text { D: } 9\end{array}$ & $\begin{array}{l}\text { T7-S5, ABC: } 13 \\
\text { D: } 3\end{array}$ & \\
\hline \multicolumn{4}{|l|}{ Comorbidities } \\
\hline $\begin{array}{l}\text { Diagnosed sleep disordered } \\
\text { breathing^ }\end{array}$ & $25(49 \%)$ & $4(12 \%)$ & $0(0 \%)$ \\
\hline Current smoking $\wedge$ & $10(20 \%)$ & $9(27 \%)$ & $3(6 \%)$ \\
\hline History of hypertension ${ }^{\wedge}$ & $3(6 \%)$ & $5(15 \%)$ & $0(0 \%)$ \\
\hline Diabetes mellitus & $3(6 \%)$ & $2(6 \%)$ & $0(0 \%)$ \\
\hline Ischaemic heart disease & $2(4 \%)$ & $2(6 \%)$ & $0(0 \%)$ \\
\hline Cerebrovascular disease & $2(4 \%)$ & $0(0 \%)$ & $0(0 \%)$ \\
\hline Dyslipidaemia & $2(4 \%)$ & $1(3 \%)$ & $2(4 \%)$ \\
\hline Atrial fibrillation & $0(0 \%)$ & $0(0 \%)$ & $1(2 \%)$ \\
\hline
\end{tabular}

$S C I$ spinal cord injury, $A B C$ American Spinal Injury Association Impairment Scale (AIS) A, B, or C, D AIS D

${ }^{*} p<0.05$ compared with controls; $\wedge^{\wedge} p<0.05$ across groups. Data given as mean \pm s.d

reverse dipper category for the complete tetraplegia group, but in the nondipper category for the incomplete tetraplegia group (Table 3). The betweengroup difference in night:day SBP of $7.2 \%$ (95\% CI, -0.1 to 14.5 ), as determined in pairwise comparisons, suggests a trend towards a more abnormal diurnal $\mathrm{BP}$ variation for the complete compared with incomplete tetraplegia group. Reverse dipping was observed in 60 and $33 \%$ of complete and incomplete tetraplegia, respectively, while dipping was observed in 15 and $33 \%$, respectively. Completeness of injury was not associated with statistically significant differences in BP or pulse rate parameters in paraplegia or in tetraplegia.

\section{Postural BP}

SBP and DBP were slightly higher after postural change in control participants, but in both tetraplegia and paraplegia, BP was lower (Fig. 1). Orthostatic hypotension was present in $38 \%(n=10), 25 \%(n=4)$, and $6 \%(n=2)$ of participants with tetraplegia or paraplegia and the controls, respectively $(p<0.05)$.

\section{Urine production}

Urine flow rates were statistically lower at night than during the day in controls and in participants with paraplegia, but this nocturnal fall in urine flow rate was not observed in the participants with tetraplegia (Fig. 2a).

In participants with SCI, the nocturnal fall in urine production was present in those with a dipper BP pattern, in contrast to the reversal of diurnal urine production in the reverse dipper group (Fig. 2b). In participants with tetraplegia, the nocturnal fall in urine production was absent in all groups, but a reversal of diurnal urine production was observed in the group with the reverse dipper pattern (Fig. 2c). In participants with complete SCI, the nocturnal fall in urine production was present only in those with the dipper BP pattern (Fig. 2d).

No correlation was observed between night:day SBP and night:day urine flow rates.

\section{Symptom scores}

Symptoms of orthostatic intolerance were greatest in participants with tetraplegia and lowest in controls (Fig. 3). 
Table 2 Blood pressure and pulse rate for all participants

\begin{tabular}{lllll}
\hline & & Tetraplegia $(n=47)$ & Paraplegia $(n=30)$ & Controls $(n=51)$ \\
\hline Systolic BP (mmHg) & $24 \mathrm{~h}$ & $110.8 \pm 1.5^{* * \dagger \dagger}$ & $119.4 \pm 2.1$ & $118.1 \pm 1.4$ \\
& Day & $111.5 \pm 1.9^{* * \dagger \dagger}$ & $125.3 \pm 2.2$ & $124.1 \pm 1.5$ \\
& Night & $108.2 \pm 1.7$ & $109.6 \pm 2.8$ & $106.9 \pm 1.4$ \\
& Night:Day $(\%)$ & $97.8 \pm 1.6^{* * \dagger \dagger}$ & $87.6 \pm 1.8$ & $86.2 \pm 0.8$ \\
Reverse dippers & & $21(45 \%)$ & $4(13 \%)$ & $1(2 \%)^{\wedge}$ \\
Diastolic BP (mmHg) & $24 \mathrm{~h}$ & $64.4 \pm 1.2^{* * \dagger \dagger}$ & $69.8 \pm 1.5$ & $69.8 \pm 1.0$ \\
& Day & $66.3 \pm 1.3^{* * \dagger \dagger}$ & $74.9 \pm 1.6$ & $75.0 \pm 1.1$ \\
Hypertension & Night & $60.7 \pm 1.2$ & $61.4 \pm 1.8$ & $59.2 \pm 1.2$ \\
& $24 \mathrm{~h}$ & $2(4 \%)$ & $5(17 \%)$ & $11(22 \%)^{\wedge}$ \\
Pulse rate (bpm) & Day & $2(4 \%)$ & $5(17 \%)$ & $13(25 \%)^{\wedge}$ \\
& Night & $6(13 \%)$ & $7(23 \%)$ & $9(18 \%)$ \\
& $24 \mathrm{~h}$ & $65.2 \pm 1.5^{* * \dagger \dagger}$ & $78.1 \pm 1.7 *$ & $71.3 \pm 1.2$ \\
& Day & $68.4 \pm 1.5^{* * \dagger \dagger}$ & $83.2 \pm 1.9 *$ & $75.9 \pm 1.4$ \\
\end{tabular}

$B P$ blood pressure

${ }^{*} p<0.05$ vs. controls; ${ }^{* *} p<0.01$ vs. controls; ${ }^{\dagger} p<0.05$ vs. paraplegia; ${ }^{\dagger \dagger} p<0.01$ vs. paraplegia; ${ }^{\wedge} p<0.05$ across groups. Values are mean \pm s.e.m

Table 3 Blood pressure and pulse rates for complete and incomplete tetraplegia and paraplegia

\begin{tabular}{|c|c|c|c|c|c|}
\hline & & $\begin{array}{l}\text { Tetraplegia, complete } \\
(n=20)\end{array}$ & $\begin{array}{l}\text { Tetraplegia, incomplete } \\
(n=27)\end{array}$ & $\begin{array}{l}\text { Paraplegia, complete } \\
(n=23)\end{array}$ & $\begin{array}{l}\text { Paraplegia, incomplete } \\
(n=7)\end{array}$ \\
\hline \multirow{4}{*}{$\begin{array}{l}\text { Systolic BP } \\
(\mathrm{mmHg})\end{array}$} & $24 \mathrm{~h}$ & $108.6 \pm 1.5^{* * * \neq}$ & $112.4 \pm 2.4^{* *}$ & $120.1 \pm 2.5$ & $117.4 \pm 4.2$ \\
\hline & Day & $107.0 \pm 2.2 * * * \neq$ & $114.8 \pm 2.6^{* *}$ & $125.9 \pm 2.7$ & $123.6 \pm 3.9$ \\
\hline & Night & $108.2 \pm 1.7$ & $108.3 \pm 2.7$ & $110.2 \pm 3.1$ & $107.7 \pm 6.7$ \\
\hline & $\begin{array}{l}\text { Night: } \\
\text { Day }(\%)\end{array}$ & $101.9 \pm 2.4^{* * * t+}$ & $94.8 \pm 2.1 * *$ & $87.7 \pm 2.0$ & $87.1 \pm 4.3$ \\
\hline Reverse dippers & & $12(60 \%)$ & $9(33 \%)$ & $2(9 \%)$ & $2(29 \%)^{\wedge}$ \\
\hline \multirow{3}{*}{$\begin{array}{l}\text { Diastolic BP } \\
(\mathrm{mmHg})\end{array}$} & $24 \mathrm{~h}$ & $63.5 \pm 1.4^{* * \ddagger}$ & $65.1 \pm 1.7 * *$ & $70.2 \pm 1.9$ & $68.6 \pm 2.2$ \\
\hline & Day & $63.8 \pm 1.7 * *+\neq$ & $68.2 \pm 1.9^{* *}$ & $75.3 \pm 2.0$ & $73.7 \pm 2.0$ \\
\hline & Night & $61.1 \pm 1.5$ & $60.5 \pm 1.7$ & $61.9 \pm 2.1$ & $59.6 \pm 3.6$ \\
\hline \multirow[t]{3}{*}{ Hypertension } & $24 \mathrm{~h}$ & $0(0 \%)$ & $2(7 \%)$ & $4(17 \%)$ & $1(14 \%)$ \\
\hline & Day & $0(0 \%)$ & $2(7 \%)$ & $4(17 \%)$ & $1(14 \%)$ \\
\hline & Night & $2(10 \%)$ & $4(15 \%)$ & $5(22 \%)$ & $2(29 \%)$ \\
\hline \multirow[t]{3}{*}{ Pulse rate (bpm) } & $24 \mathrm{~h}$ & $63.3 \pm 1.9 *$ & $66.7 \pm 2.2^{\dagger}$ & $77.9 \pm 2.1$ & $78.8 \pm 2.8$ \\
\hline & Day & $67.0 \pm 2.0^{*}$ & $69.4 \pm 2.1^{\dagger}$ & $83.1 \pm 2.4$ & $83.2 \pm 2.5$ \\
\hline & Night & $58.0 \pm 1.6^{\ddagger \ddagger}$ & $62.3 \pm 2.4$ & $69.2 \pm 2.2$ & $68.7 \pm 4.3$ \\
\hline
\end{tabular}

$B P$ blood pressure, bpm beats per minute

Data for control participants shown in Table 2

${ }^{*} p<0.05$ vs. controls; ${ }^{* *} p<0.01$ vs. controls; ${ }^{\dagger} p<0.05$ vs. incomplete paraplegia; ${ }^{\dagger \dagger} p<0.01$ vs. incomplete paraplegia; ${ }^{\ddagger} p<0.05$ vs. complete paraplegia; ${ }^{\dagger} p<0.01$ vs. complete paraplegia; ${ }^{\wedge} p<0.05$ across groups; Values are mean \pm s.e.m

Scores were not significantly different between high and low paraplegia, complete and incomplete tetraplegia, or complete and incomplete paraplegia (data not shown). There was no significant difference in symptom scores among the three diurnal BP categories (dipper, nondipper, and reverse dipper).

\section{Discussion}

We previously documented the haemodynamic consequences of acute SCI by studying a large group of participants recruited from consecutive admissions to a singlesite unit that had responsibility for treating people with 

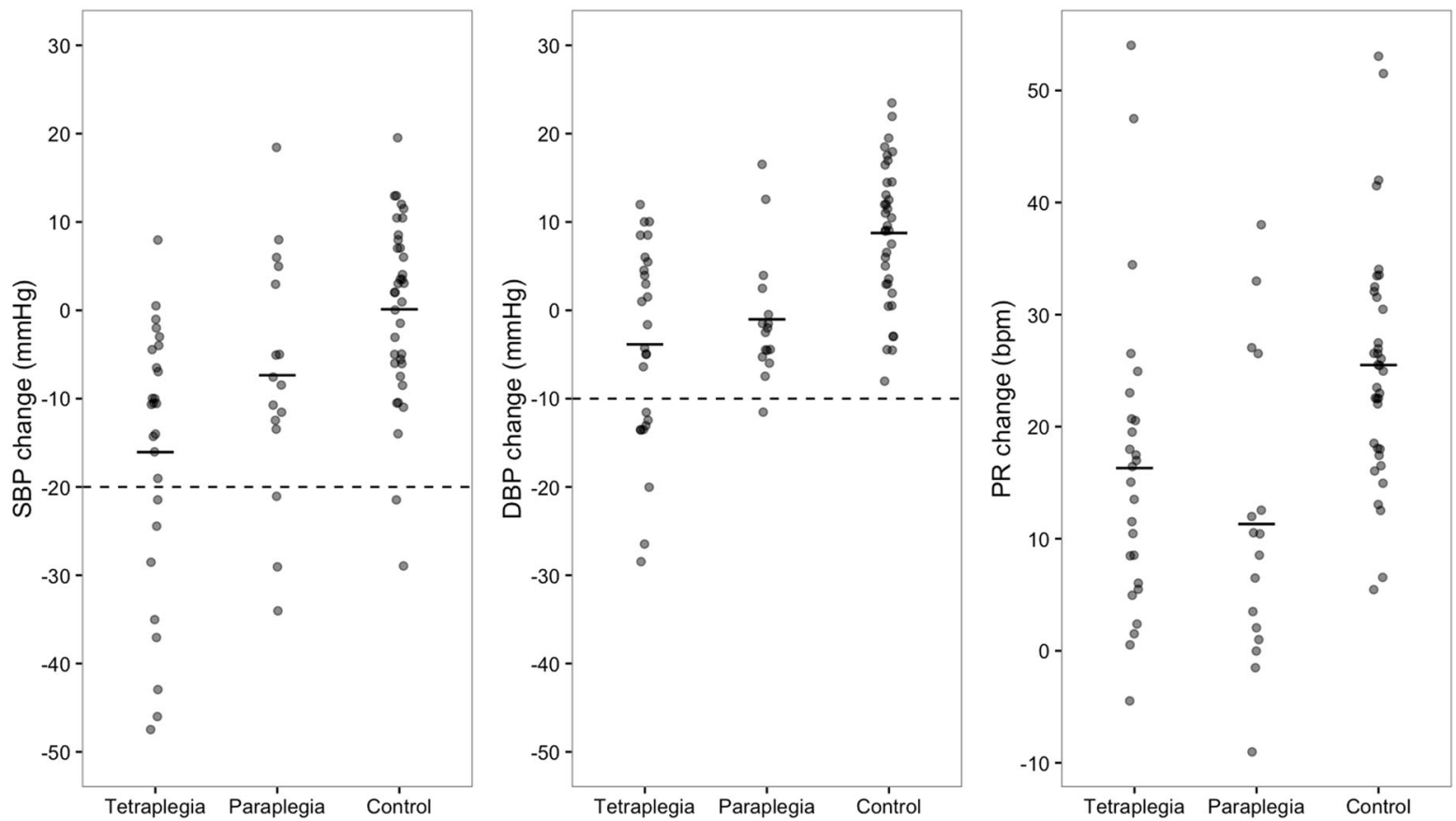

Fig. 1 Postural changes in blood pressure and pulse rates for tetraplegia $(n=26)$, paraplegia $(n=16)$, and controls $(n=36)$. Dashed line indicates limit for orthostatic hypotension. Solid short lines

represent mean. SBP systolic blood pressure, DBP diastolic blood pressure, PR pulse rate, bpm beats per minute
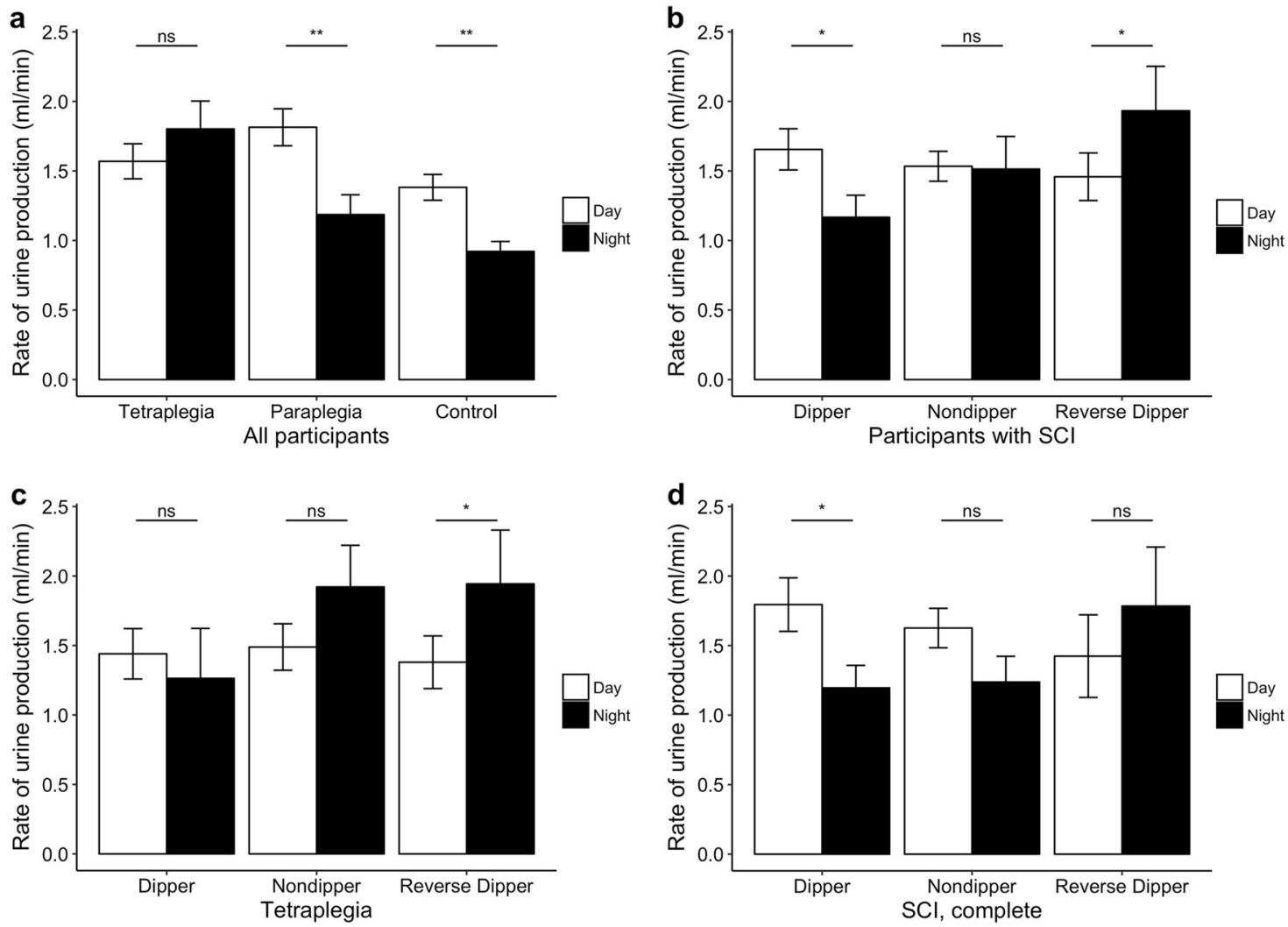

Fig. 2 a Day and night urine flow rates for tetraplegia $(n=31)$, paraplegia $(n=24)$, and controls $(n=42)$. b Urine flow rates for participants with SCI and dipper $(n=19)$, nondipper $(n=13)$, and reverse dipper $(n=16)$ patterns. c Urine flow rates for participants with tetraplegia and dipper $(n=7)$, nondipper $(n=8)$, and reverse

dipper $(n=12)$ patterns. d Urine flow rates for participants with complete SCI and dipper $(n=11)$, nondipper $(n=9)$, and reverse dipper $(n=7)$ patterns. Error bars represent s.e.m. Significance values above each pair of bars indicate comparison between day vs. night rate; ns not significant; ${ }^{*} p<0.05 ;{ }^{* *} p<0.01$ 


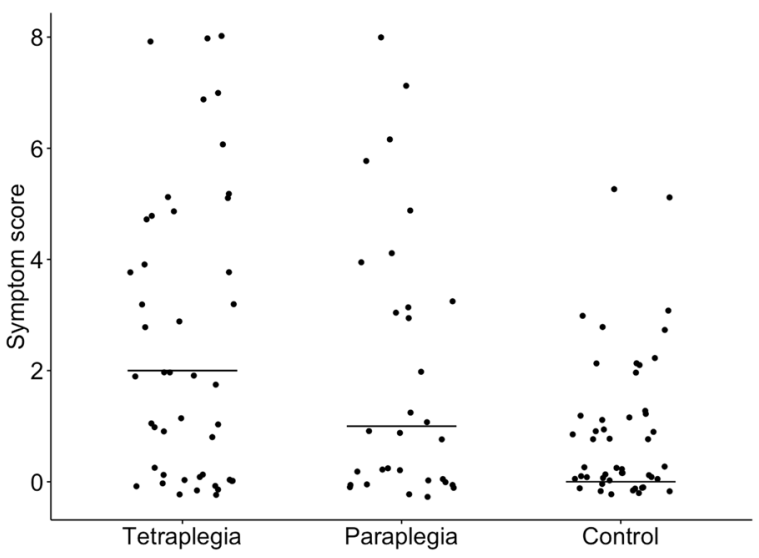

Fig. 3 Orthostatic intolerance symptom scores for tetraplegia $(n=45)$, paraplegia $(n=31)$, and controls $(n=48)$. Horizontal lines represent median scores. Chi-squared $=8.94, \mathrm{df}=2, p=0.0114$

traumatic SCI from a defined region of Australia [3]. The present study aimed to examine the effects of SCI on BP over the longer term. Similar to our previous study and in contrast to other investigations [12-16], we examined a large cohort of participants and employed few exclusion criteria. Also, diurnal BP was categorised according to patterns associated with poorer cardiovascular outcomes in the general population $[9,17]$.

We previously found that acute SCI disrupts diurnal variation of BP in $94 \%$ of participants with tetraplegia and $65 \%$ of those with paraplegia and these changes largely persisted over the 12 months after cervical injury, but they tended to improve in paraplegia [3]. In this study of participants with a longer-term injury, we again observed a high prevalence of abnormal diurnal BP variation amongst participants with tetraplegia (74\%). However, abnormal diurnal BP variation was only seen in $30 \%$ of participants with long-term paraplegia, compared with $18 \%$ in the control population. This apparent time-dependent improvement in BP control following paraplegic injury is consistent with findings in animal models of mid-thoracic SCI in which diurnal BP variation returned after 5 days following incomplete T5 injury [18] and after 14-21 days following complete T3 injury [19]. Taken together, the results of these studies suggest that SCI causes substantial disruption to diurnal BP control which largely persists following tetraplegic injury, but which improves after paraplegic injury.

The return of diurnal BP variation in paraplegia could potentially be dependent on preservation of sympathetic inputs to the spinal cord below $\mathrm{C} 8$. The sympathetic outputs between T1-T6 regulate orthostatic vascular responses, and the severity of cardiovascular dysfunction following SCI has previously been correlated with the extent of histopathological changes in the vasomotor pathways in the spinal cord [20]. Consistent with a neurological explanation is that participants with an incomplete tetraplegic injury had less abnormalities in diurnal variation of BP than participants with a complete injury. Also consistent with this hypothesis is the higher pulse rates observed in the paraplegic group, particularly in low paraplegia. In contrast, the pulse rate in tetraplegic participants was lower than in controls. This finding is similar to our observation following acute injury, thus it appears that the elevation in pulse rates following paraplegia begins soon after SCI and persists in the longer term. This observation would be consistent with paraplegia being in a state of increased activation of the sympathetic nervous system, and perhaps that may explain why their BP is similar to that of the controls. In contrast, in tetraplegia it appears that the ability to mount an adrenergic response has been lost.

Consistent with our previous studies [21], we found a high prevalence of sleep disordered breathing (SDB) among people with tetraplegia. Obstructive sleep apnoea, the predominant form of SDB [21], has been associated with abnormal diurnal BP variation in the able-bodied population [22] and therefore might have contributed to the BP abnormalities observed in this study. It was unfortunately outside the scope of this study to measure sleep indices on all participants, which would have allowed for a comparison of effects on sleep disturbances, including effects of any management for SDB, on diurnal BP. The lack of precision in our estimates of SDB preclude any meaningful interpretation of these differences.

The increased incidence of cardiovascular disease in SCI mandates strict control of cardiovascular risk factors. Compared with clinic measurements, ambulatory BP better predicts cardiovascular risk [23]. Although the predictive powers of ambulatory pressure have not been studied in SCI, in our population of relatively unselected participants, measurement of ambulatory pressure revealed that $17 \%$ $(n=13)$ of our SCI participants had nocturnal hypertension, but in only $5 \%(n=4)$ did an elevated day pressure provide a clue about the high night pressure. While these data argue for the use of ambulatory measurements in the SCI population, it should be noted that the results of ambulatory monitoring only triggered antihypertensive treatment in one of our study participants-at least in part because relatively low day pressures produced a 24-h average that was below treatment thresholds.

Nocturnal nondipping involves the loss of decline in night BP relative to day pressure, whereas nocturnal hypertension is defined when average night BP exceeds an absolute value, thus one may occur independently of the other. We observed a high prevalence of nondipping in people with tetraplegia. While the prevalence of nocturnal hypertension was not different between SCI and controls, it was not insignificant, considering that people with longterm SCI have been observed to have a higher rate of other cardiovascular risk factors [2], which contributes to higher 
absolute cardiovascular risk. Nondipping and nocturnal hypertension have independently been associated with poorer outcomes in normotensive as well as hypertensive able-bodied populations [23, 24]. Their impact on cardiovascular risk, and whether treatment to normalise them will improve outcomes in people with SCI, remains to be investigated.

Although orthostatic hypotension may be asymptomatic in SCI, it can also manifest as lightheadedness, fatigue, and syncope $[1,25]$. Symptoms can affect activities of daily living, delay rehabilitation and impair quality of life $[1,25$, 26]. In our previous study of a large group of unselected participants with SCI, we found a high prevalence of symptoms, particularly in those with complete cervical injury, and these were only partly improved 12 months later. Whether these symptoms would persist over the longer term is unclear, with previous smaller studies of selected people with SCI having provided conflicting results $[1,25]$. In the present study we observed that participants with tetraplegia continued to be symptomatic, and at higher rate than those with paraplegia, suggesting there was not a continuous improvement in orthostatic symptoms beyond 12 months after injury. It should also be noted that although the difference in median values between those with tetraplegia and controls was relatively small, this is not to say symptoms were insignificant. Some individuals reported severe symptoms even many years after their injury (Fig. 3).

As in our previous study, we measured diurnal urine production in people with SCI. Nocturnal polyuria in SCI has been attributed to the loss of vascular tone due to impaired autonomic function, alongside lack of the skeletal muscle pump, resulting in lower limb venous pooling while upright; recumbency overnight returns circulating volume thus increasing renal perfusion pressures, which, together with a lack of nocturnal increase in antidiuretic hormone [16], leads to nocturnal diuresis. Other hormonal changes such as rises in atrial natriuretic peptide and glucocorticoid, and suppression of the renin-angiotensin-aldosterone system, have also been implicated in post-SCI polyuria in human and animal studies [27, 28]. There is also evidence for an interaction of neural networks involved in the control of the bladder function and lower limb musculature. Locomotor training, including training limited to the forelimbs, has been found to reduce post-SCI polyuria in rats. It also been associated with improvements in bladder and urinary tract function in rat studies, in keeping with a few reports in humans with SCI [29].

In previous studies [3, 16], diurnal variation of urine production was lost in both tetraplegia and paraplegia. In contrast, and within the limits of the data from our urinary measures, we observed a nocturnal fall in urine output in both high and low paraplegia and in complete paraplegia. This may be related to time after SCI as previous studies were done on participants admitted for acute SCI or undergoing inpatient rehabilitation following admission for SCI. The compensatory neuro-hormonal mechanisms occurring over time that affect diurnal BP, described above, may also affect diurnal urine production. The ability to maintain daytime BP in paraplegia may reduce the fluid shifts seen in tetraplegia that result in nocturnal diuresis. Another factor could be the effect of physical activity over time, that leads to the changes in diurnal urine production, by its effect on the urinary tract and bladder function, as described in the rat models of thoracic SCI above.

Relative elevation of supine BP consequent upon SCI has been proposed as a cause or contributor to a nocturnal diuresis and even potentially to orthostatic hypotension $[4$, 5]. That hypothesis is supported by the results of the current study in which chronic tetraplegia was associated with nocturnal nondipping and diuresis. Also consistent with this hypothesis was the observation that the improvement in diurnal BP variation in participants with paraplegia was accompanied by a normalisation of the diurnal production of urine. However, in the current trial, we did not find that the improvements in these parameters in the participants with paraplegia were accompanied by an improvement in orthostatic symptoms. That being said, this trial was not designed or powered to detect such a change-and perhaps the only way of testing this hypothesis would be to determine the symptomatic effects of selectively altering nocturnal BP or diuresis.

Limitations of our study include that it examined a sample of convenience, with the population being recruited at a single centre, and willing to participate, thus findings may not be generalisable to the greater SCI population.

\section{Conclusions}

This study has found a high prevalence of abnormal diurnal variation in BP and urine output, and of orthostatic hypotension and symptoms, in tetraplegia compared with paraplegia and controls. While nocturnal hypertension was not different in prevalence between SCI and controls, daytime hypertension was lower in tetraplegia. ABPM can be used to identify these BP patterns, which are associated with poorer prognoses in the general population, and are potential contributing factors to the observed increased prevalence of cardiovascular disease in the SCI population.

Acknowledgements This research was supported by a grant from the Transport Accident Commission (DP174). MG is supported by a scholarship and stipend through the University of Melbourne and Spinal Research Institute. We thank Dr. Sue Finch and the Statistical Consulting Centre, University of Melbourne, for their assistance in statistical analysis. 


\section{Compliance with ethical standards}

Conflict of interest The authors declare that they have no conflict of interest.

\section{References}

1. Mathias CJ, Low DA, Frankel HL. Autonomic disturbances in spinal cord lesions. In: Mathias CJ, Bannister R, editors. Autonomic failure: a textbook of clinical disorders of the autonomic nervous system. 5th ed. Oxford: Oxford University Press; 2013. pp. 797-817.

2. Myers J, Lee M, Kiratli J. Cardiovascular disease in spinal cord injury: an overview of prevalence, risk, evaluation, and management. Am J Phys Med Rehabil. 2007;86:142-52.

3. Goh MY, Millard MS, Wong ECK, Brown DJ, Frauman AG, O'Callaghan CJ. Diurnal blood pressure and urine production in acute spinal cord injury compared with controls. Spinal Cord. 2017;55:39-46.

4. Mathias CJ. Orthostatic hypotension and paroxysmal hypertension in humans with high spinal cord injury. Prog Brain Res. 2006;152:231-43.

5. Omboni S, Smit AA, van Lieshout JJ, Settels JJ, Langewouters GJ, Wieling W. Mechanisms underlying the impairment in orthostatic tolerance after nocturnal recumbency in patients with autonomic failure. Clin Sci. 2001;101:609-18.

6. DeVivo MJ, Biering-Sørensen F, New P, Chen Y. Standardization of data analysis and reporting of results from the International Spinal Cord Injury Core Data Set. Spinal Cord. 2011;49:596-9.

7. O'Brien E, Asmar R, Beilin L, Imai Y, Mallion J-M, Mancia G, et al. European Society of Hypertension recommendations for conventional, ambulatory and home blood pressure measurement. J Hypertens. 2003;21:821-48.

8. Fagard R, Brguljan J, Thijs L, Staessen J. Prediction of the actual awake and asleep blood pressures by various methods of $24 \mathrm{~h}$ pressure analysis. J Hypertens. 1996;14:557-63.

9. Ohkubo T, Imai Y, Tsuji I, Nagai K, Watanabe N, Minami N, et al. Relation between nocturnal decline in blood pressure and mortality. The Ohasama Study. Am J Hypertens. 1997;10:1201-7.

10. Head GA, McGrath BP, Mihailidou AS, Nelson MR, Schlaich MP, Stowasser M, et al. Ambulatory blood pressure monitoring in Australia: 2011 consensus position statement. J Hypertens. 2012;30:253-66.

11. Freeman R, Wieling W, Axelrod FB, Benditt DG, Benarroch E, Biaggioni I, et al. Consensus statement on the definition of orthostatic hypotension, neurally mediated syncope and the postural tachycardia syndrome. Clin Auton Res. 2011;21:69-72.

12. Krum H, Louis WJ, Brown DJ, Jackman GP, Howes LG. Diurnal blood pressure variation in quadriplegic chronic spinal cord injury patients. Clin Sci. 1991;80:271-6.

13. Nitsche B, Perschak H, Curt A, Dietz V. Loss of circadian blood pressure variation in complete tetraplegia. J Hum Hypertens. 1996;10:311-7.

14. Munakata M, Kameyama J, Kanazawa M, Nunokawa T, Moriai N, Yoshinaga K. Circadian blood pressure rhythm in patients with higher and lower spinal cord injury: simultaneous evaluation of autonomic nervous activity and physical activity. J Hypertens. 1997;15:1745-9.

15. Rosado-Rivera D, Radulovic M, Handrakis JP, Cirnigliaro CM, Jensen AM, Kirshblum S, et al. Comparison of 24-hour cardiovascular and autonomic function in paraplegia, tetraplegia, and control groups: implications for cardiovascular risk. J Spinal Cord Med. 2011;34:395-403.

16. Kilinç S, Akman MN, Levendoglu F, Ozker R. Diurnal variation of antidiuretic hormone and urinary output in spinal cord injury. Spinal Cord. 1999;37:332-5.

17. Verdecchia P, Schillaci G, Borgioni C, Ciucci A, Gattobigio R, Guerrieri $\mathrm{M}$, et al. Altered circadian blood pressure profile and prognosis. Blood Press Monit. 1997;2:347-52.

18. Mayorov DN, Adams MA, Krassioukov AV. Telemetric blood pressure monitoring in conscious rats before and after compression injury of spinal cord. J Neurotrauma. 2001;18:727-36.

19. West CR, Popok D, Crawford MA, Krassioukov AV. Characterizing the temporal development of cardiovascular dysfunction in response to spinal cord injury. J Neurotrauma. 2015;32:922-30.

20. Furlan JC, Fehlings MG, Shannon P, Norenberg MD, Krassioukov AV. Descending vasomotor pathways in humans: correlation between axonal preservation and cardiovascular dysfunction after spinal cord injury. J Neurotrauma. 2003;20:1351-63.

21. Berlowitz J, Spong J, Gordon I, Howard E, Brown J. Relationships between objective sleep indices and symptoms in a community sample of people with tetraplegia. Arch Phys Med Rehabil. 2012;93:1246-53.

22. Wolf J, Hering D, Narkiewicz K. Non-dipping pattern of hypertension and obstructive sleep apnea syndrome. Hypertens Res. 2010;33:867-71.

23. Dolan E, Stanton A, Thijs L, Hinedi K, Atkins N, McClory S, et al. Superiority of ambulatory over clinic blood pressure measurement in predicting mortality: the Dublin outcome study. Hypertension. 2005;46:156-61.

24. Fan H-Q, Li Y, Thijs L, Hansen TW, Boggia J, Kikuya M, et al. Prognostic value of isolated nocturnal hypertension on ambulatory measurement in 8711 individuals from 10 populations. J Hypertens. 2010;28:2036-45.

25. Claydon VE, Krassioukov AV. Orthostatic hypotension and autonomic pathways after spinal cord injury. J Neurotrauma. 2006;23:1713-25

26. Illman A, Stiller K, Williams M. The prevalence of orthostatic hypotension during physiotherapy treatment in patients with an acute spinal cord injury. Spinal Cord. 2000;38:741-7.

27. Denys M-A, Viaene A, Goessaert A-S, Van Haverbeke F, Hoebeke P, Raes A, et al. Circadian rhythms in water and solute handling in adults with a spinal cord injury. J Urol. 2016;197:445-51.

28. Montgomery LR, Hubscher $\mathrm{CH}$. Altered vasopressin and natriuretic peptide levels in a rat model of spinal cord injuryimplications for the development of polyuria. Am J Physiol Renal Physiol. 2017. https://doi.org/10.1152/ajprenal.00229.2017.

29. Hubscher $\mathrm{CH}$, Montgomery LR, Fell JD, Armstrong JE, Poudyal $\mathrm{P}$, Herrity AN, et al. Effects of exercise training on urinary tract function after spinal cord injury. Am J Physiol Renal Physiol. 2016;310:F1258-68. 